\title{
Water/methanol Repellency and Preparation of Biomimetic Silver Film on Butterfly Wing
}

\author{
Yan Fang ${ }^{\mathrm{a}}$, Gang Sun ${ }^{\mathrm{b}^{*}}$, Wanxing Wang ${ }^{\mathrm{c}}$ and Jingshi Yin ${ }^{\mathrm{d}}$ \\ School of Life Science, Changchun Normal University, Changchun 130032, China \\ afangyan124@aliyun.com, bsungang@nenu.edu.cn, c1481399783@qq.com, d634229991@qq.com
}

\begin{abstract}
The hydrophobicity and oleophobicity (methanol repellency) of butterfly wing surfaces were measured by a video-based contact angle (CA) meter. The multi-dimensional microstructure of the wing surfaces was characterized by a scanning electron microscope (SEM) and an atomic force microscope (AFM). The wing surface exhibits superhydrophobicity (water CA $150.8 \sim 158.5^{\circ}$ ) and low adhesion (water sliding angle $1 \sim 4^{\circ}$ ). Meanwhile, the wing surface displays high repellency against methanol. The critical concentrations for wetting and spreading-wetting of methanol solution on the wing surface are $60 \%$ and $85 \%$, respectively. The wing surface is of hydro-oleophobicity. The wing surface possesses complicated hierarchical microstructures. Using the butterfly wing as a bio-template, the hydrophobic silver films were prepared. Water CA increases from metal silver's intrinsic CA $63.0^{\circ}$ to up to $140.6^{\circ}$ (Speyeria aglaja, $5 \mathrm{~nm}$ silver film). The micro-morphology on the wing result in the transition of metal silver from hydrophilic to hydrophobic. The butterfly wing can be used as a template for design of smart interface and functional surface.
\end{abstract}

Keywords: Superhydrophobicity, Oleophobicity, Preparation, Metal film, Biomaterial.

\section{Introduction}

After millions of years of evolution, many creatures have possessed peculiar body surfaces which are superhydrophobic, self-cleaning, anti-adhesive, drag reducing, self-healing, anti-wearing and so on [1]. In the last few years, more and more researches have been focused on fabrication of materials with special properties and functions using biological surfaces as templates. The special functional surfaces with complex wettability, such as amphilicity (i.e. hydrophilic-lipophilic properties), hydro-oleophobicity (i.e. water-oil repellency), hydrophilic and oleophobic properties, hydrophobic-lipophilic properties, are often needed in engineering domain [2]. Butterfly wing is one of the most complicated three-dimensional periodical substrates in nature [3]. In this paper, the hydrophobicity and oleophobicity (methanol repellency) of butterfly wing surfaces were measured. Using the butterfly wing as a bio-template, hydrophobic silver films were fabricated. The results may provide evidence for preparation of special functional surface used in harsh industrial environments, as well as enlightenment for biomimetic design of superhydro-oleophobic surface.

\section{Materials and Methods}

\subsection{Materials}

The specimens of ten butterfly species were collected in Changchun City. The wings were cleaned, desiccated and flattened, then cut into $5 \mathrm{~mm} \times 5 \mathrm{~mm}$ pieces from discal cell (Fig. 1). The reagents used were of analytical reagents (AR) grade and purchased from Tianjin Pharmaceuticals Group Co. Ltd., China. The volume of water droplet for CA and sliding angle (SA) measurements was $5 \mu 1$.

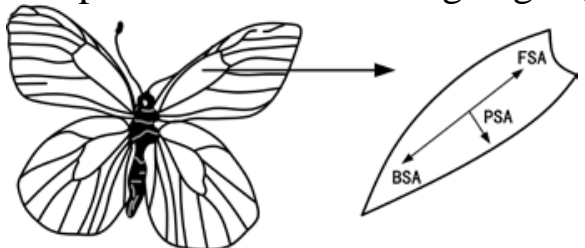

Fig. 1 The experimental area and the sliding angles in various directions on the butterfly wing 


\subsection{Measurements of Wetting Angles}

Using an optical CA measuring system (DataPhysics OCA20, Germany), the CAs of water and methanol solution on the wing surface were measured via sessile drop method at ambient temperature of $25 \pm 1{ }^{\circ} \mathrm{C}$. The SA of water droplets was measured along three different directions, including forward SA (FSA, the SA of droplet from wing base to wing terminal end), backward SA (BSA, the SA of droplet from wing terminal end to wing base), and perpendicular SA (PSA, the SA of droplet perpendicular to the major axis of wing) (Fig. 1).

\subsection{Characterization of Micro/nano-structure}

After gold coating by an ion sputter coater (Hitachi E-1045, Japan), the wing pieces were observed and photographed by a SEM (Hitachi SU8010, Japan) and an AFM (Bruker Dimension Icon, USA).

\subsection{Biomimetic Fabrication of Hydrophobic Silver Film}

A vacuum vapor coating machine (OLED300D, Shenyang Scientists Friend Vacuum Technology Company, China) was used for coating of silver film on the wing surface. The thickness of silver film was determined as follows:

$$
\Delta H=\frac{\rho \times d}{0.1329}
$$

where, $\rho$ is the density of metal $\left(\mathrm{g} / \mathrm{cm}^{3}\right), d$ is the thickness of metal film coated $(\mathrm{nm}), \Delta H$ is the change in excitation frequency $(\mathrm{Hz})$. For metal silver, $\rho_{\mathrm{Ag}}=10.5 \mathrm{~g} / \mathrm{cm}^{3}$. The thicknesses of silver films coated on the wing surface were $5,10,20,40,60,80,100 \mathrm{~nm}$, respectively, meanwhile the silver films of the same thicknesses were coated on glass slides as contrast.

\section{Results and Discussion}

\subsection{Water and Methanol Repellency of the Wing Surface}

The wing surfaces of the ten butterfly species are superhydrophobic (CA 150.8 158.5 ${ }^{\circ}$ ), the average value is $153.2^{\circ}$. Meanwhile, the butterfly wing surfaces have low adhesion, the water SA is extremely small (FSA $1 \sim 4^{\circ}$, BSA $4 \sim 11^{\circ}$, PSA $3 \sim 7^{\circ}$ ). The butterfly wing surface is of low adhesive superhydrophobicity. There are significant differences between the water SAs in various directions $(p<0.01)$, FSA $<$ PSA $<$ BSA (Table 1). The sliding behavior of a droplet on the wing surface shows remarkable anisotropic property. Even a very slight tilting $\left(1 \sim 4^{\circ}\right)$ of the wing is sufficient to cause the water droplet to roll off readily and take away the contaminating particles efficiently [4].

Table 1 Contact angle and sliding angle of water, the minimum (critical) concentrations for wetting and spreading-wetting of methanol solution on the butterfly wing surfaces

\begin{tabular}{|cccccccc|}
\hline \multirow{2}{*}{ Species } & \multicolumn{2}{c}{ Water CA $\left(^{\circ}\right)$} & Water SA $\left(^{\circ}\right)$ & $\begin{array}{c}\text { The minimum concentrations for } \\
\text { wetting and spreading-wetting of } \\
\text { methanol solution (\%) }\end{array}$ \\
\cline { 2 - 9 } & $\begin{array}{c}\text { With } \\
\text { Scale }\end{array}$ & $\begin{array}{c}\text { Without } \\
\text { Scale }\end{array}$ & FSA & BSA & PSA & $\begin{array}{c}\text { Wetting } \\
\left(\text { CA }<90^{\circ}\right)\end{array}$ & $\begin{array}{c}\text { Spreading-wetting } \\
\left(\mathrm{CA} \rightarrow 0^{\circ}\right)\end{array}$ \\
\hline Anthocharis cardamines & 153.4 & 122.1 & 3 & 11 & 4 & 65 & 90 \\
\hline Colias fieldii & 152.7 & 121.3 & 2 & 7 & 6 & 60 & 85 \\
\hline Euchloe ausonia & 158.5 & 117.9 & 1 & 4 & 5 & 60 & 85 \\
\hline Hyponephele interposita & 154.6 & 110.8 & 3 & 7 & 4 & 70 & 95 \\
\hline Leptidea morsei & 151.9 & 118.6 & 2 & 5 & 5 & 65 & 95 \\
\hline Lethe marginalis & 150.8 & 116.5 & 4 & 6 & 3 & 60 & 85 \\
\hline Ninguta schrenkii & 153.2 & 125.4 & 3 & 7 & 5 & 60 & 85 \\
\hline Satyrus ferula & 154.1 & 114.2 & 2 & 8 & 6 & 65 & 90 \\
\hline Speyeria aglaja & 151.7 & 127.9 & 3 & 8 & 7 & 60 & 90 \\
\hline Ypthima amphithea & 151.5 & 112.3 & 1 & 9 & 5 & 65 & 95 \\
\hline Average & 153.2 & 118.7 & 2 & 7 & 5 & 63 & 90 \\
\hline
\end{tabular}

The droplet of methanol solution presents three kinds of states on the wing surface: (1) As the concentration is lower than $60 \%$, the CAs of droplet on the wing surface of the ten species are bigger 
than $90^{\circ}$, exhibiting high repellency to methanol solution. If the wing surface is inclined slightly $\left(<3^{\circ}\right)$, the droplet flows away quickly. (2) As the concentration attains $60 \%$, the wing surfaces of five species are wetted; as the concentration attains $65 \%$, the wing surfaces of another four species are wetted; as the concentration attains $70 \%$, the wing surfaces of the rest one species are wetted. (3) As the concentration attains $85 \%$, the wing surfaces of four species are spreading-wetted, the CAs decline instantly to $0^{\circ}$; as the concentration attains $90 \%$, the wing surfaces of another three species are spreading-wetted; as the concentration attains 95\%, the wing surfaces of the rest three species are spreading-wetted. The minimum concentrations for wetting and spreading-wetting of methanol solution are $60 \%$ and $85 \%$, respectively (Table 1 ).

\subsection{Micro-morphology of the Wing Surfaces}

The butterfly wing surfaces display hierarchical micro/nano structures. The micrometric scales constitute the primary structure. The shape of the scale is a rectangle. The length of the scales is $57 \sim 123 \mu \mathrm{m}$, the width is $41 \sim 68 \mu \mathrm{m}$, the spacing is $52 \sim 85 \mu \mathrm{m}$ [Fig. 2(a)]. The nano longitudinal ridges and lateral bridges on the scales constitute the secondary structure [Fig. 2(b)]. The cross-section of the longitudinal ridge is triangular [Fig. 2(c)]. The fine nano stripes on the longitudinal ridges and lateral bridges constitute the tertiary structure [Fig. 2(b)]. The hierarchical microstructure plays a crucial role in determining the wettability. If the scales are removed from the wing surface, the water droplet shifts from a sphere to a hemisphere, the CAs decrease by 23.8 43.8 ${ }^{\circ}$ (Table 1 ).
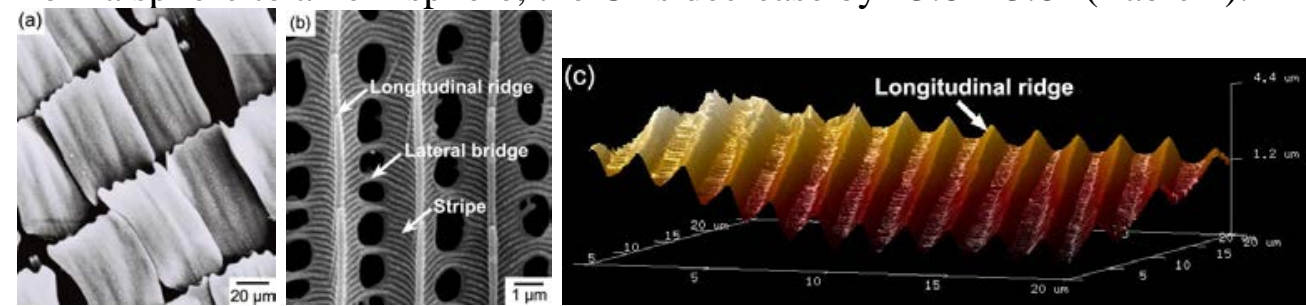

Fig. 2 The micro/nano structures of the butterfly wing surfaces

(a) primary structure (micrometric scales) (SEM); (b) secondary structure (submicrometric longitudinal ridges and lateral bridges) and tertiary structure (nano stripes) (SEM); (c) AFM image of the wing surface

\subsection{Preparation of Hydrophobic Silver Film}

As the silver film was very thin, the silver particles distribute discontinuously and irregularly [Fig. 3(a)]. With the increase of silver film thickness, the silver particles fill in the space of longitudinal ridges gradually [Fig. 3(b)]. As the silver film thickness increases to $100 \mathrm{~nm}$, the silver particles arrange uniformly and smoothly [Fig. 3(c)]. With the increase of silver film thickness, the CA on the wing surface of butterfly (Speyeria aglaja) decreases from $140.6^{\circ}$ ( $5 \mathrm{~nm}$ silver film) to $122.1^{\circ}(100$ nm silver film).
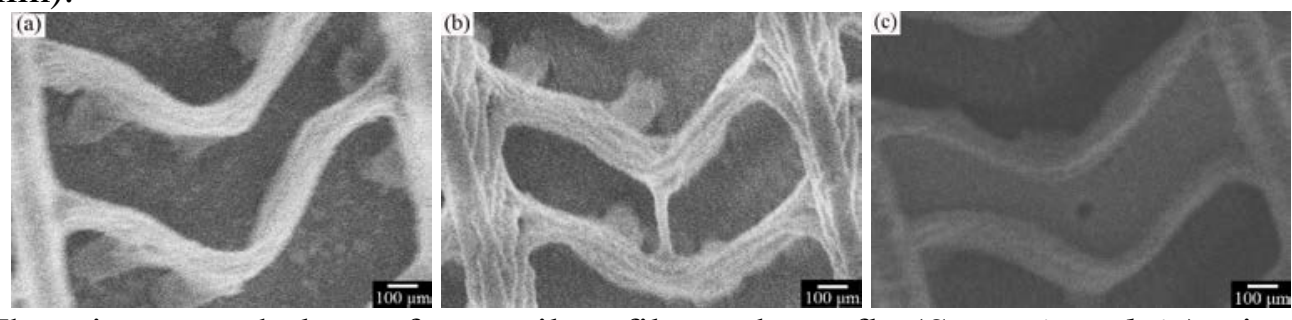

Fig. 3 The micro-morphology of nano silver film on butterfly (Speyeria aglaja) wing surface (a) $20 \mathrm{~nm}$; (b) $40 \mathrm{~nm}$; (c) $100 \mathrm{~nm}$

On the surface of glass slide coated with silver film, with the increase of silver film thickness, a smoother and smoother solid surface is formed. CAs and hydrophobicity keep decreasing: $85.1^{\circ}(5$ $\mathrm{nm}$ silver film $) \rightarrow 83.4^{\circ}(10 \mathrm{~nm}) \rightarrow 82.6^{\circ}(20 \mathrm{~nm}) \rightarrow 81.4^{\circ}(40 \mathrm{~nm}) \rightarrow 81.2^{\circ}(60 \mathrm{~nm}) \rightarrow 80.6^{\circ}(80 \mathrm{~nm})$ $\rightarrow 76.6^{\circ}(100 \mathrm{~nm})$. With the same silver film thickness, the CAs on butterfly wing surfaces are significantly greater than those on glass slide surfaces. 
As the silver film was very thin, due to the presence of primary structure and secondary structure on the wing surface, a heterogeneous contact occurs between droplet and wing surface (Cassie state), the dimension of rough structure on wing surface is much less than that of droplet. The droplet can not sink into the groove of rough surface, an "air pocket" is formed under the droplet. The apparent liquid-solid contact interface is actually composed of solid, liquid, and gas. A droplet stands upon the "air pocket”, so the surface displays superhydrophobicity (water CA $>150^{\circ}$ ). With the increase of silver film thickness, the chitin surface and rough structure on butterfly wing are covered gradually by silver film, the surface roughness decreases, a homogeneous contact occurs between droplet and nano silver film (Wenzel state). The hydrophobicity of the silver-coated surface decreases. The silver film, however, can not cover the wing surface completely. The fractal structure on the wing surface remains, the surface is still a gas-solid composite surface with micro/nano structures. The silver film decreases the surface hydrophobicity just to some extent, the wing surface displays wettability mainly of chitin rather than metal silver, the CA on the wing surface maintains over $120^{\circ}$. Metal silver is hydrophilic, with an intrinsic CA of $63.0^{\circ}$. The micro/nano hierarchical structure on the wing surface contributes to the transition of metal silver from hydrophilic to hydrophobic.

\section{Conclusions}

The surface of butterfly wing is hydro-oleophobic. The wing surface exhibits low adhesive superhydrophobicity (CA $150.8 \sim 158.5^{\circ}$, SA $1 \sim 4^{\circ}$ ) and high repellency against methanol. The critical concentrations for wetting and spreading-wetting of methanol solution on the wing surface are $60 \%$ and $85 \%$, respectively. The wing surface possesses complicated micro/nano structures, including primary structure, secondary structure and tertiary structure. Here, using butterfly wing as a natural template, an effective approach for preparation of hydrophobic nano silver film is provided. The hierarchical microstructures on the wing surface result in the transition of metal silver from hydrophilic to hydrophobic. This work can promote our understanding of wetting mechanism of bio-surfaces, and may bring useful insights into biomimetic preparation of amphiphobic surfaces, smart interfacial materials and novel self-cleaning coatings.

\section{Acknowledgments}

This work was financially supported by the National Natural Science Foundation of China (Grant No. 31671010) and the Innovative Program for Postgraduate Students of Changchun Normal University (Grant No. cscxy2015007, cscxy2017003, cscxy2017006). Dr. Prof. Gang Sun is the corresponding author of this paper.

\section{References}

[1] L. Feng, S.H. Li, Y.S. Li, H.J. Li, L.J. Zhang, J. Zhai, Y.L. Song, B.Q. Liu, L. Jiang, D.B. Zhu, Super-hydrophobic surfaces: from natural to artificial, Adv. Mater. 14 (2002) 1857-1860.

[2] G. Sun, Y. Fang, Q. Cong, L.Q. Ren, Anisotropism of the non-smooth surface of butterfly wing, J. Bion. Eng. 6 (2009) 71-76.

[3] Y. Fang, G. Sun, T.Q. Wang, Q. Cong, Hydrophobicity mechanism of non-smooth pattern on surface of butterfly wing, Chin. Sci. Bull. 52 (2007) 711-716.

[4] Y.M. Zheng, X.F. Gao, L. Jiang, Directional adhesion of superhydrophobic butterfly wings, Soft Matter 3 (2007) 178-182. 